\title{
A Case of Microangiopathic Antiphospholipid-Associated Syndromes during Pregnancy: Review of the Literature
}

\author{
Nobuhiro Suzumori, ${ }^{1,2}$ Shintaro Obayashi, ${ }^{1,2}$ Kyoko Kumagai, ${ }^{1,2}$ Shinobu Goto, ${ }^{1,2}$ \\ Atsuhiro Yoshida, ${ }^{3}$ and Mayumi Sugiura-Ogasawara ${ }^{1}$ \\ ${ }^{1}$ Department of Obstetrics and Gynecology, Nagoya City University, Graduate School of Medicine, Nagoya 467-8601, Japan \\ ${ }^{2}$ Division of Clinical and Molecular Genetics, Nagoya City University, Graduate School of Medicine, Nagoya 467-8601, Japan \\ ${ }^{3}$ Department of Cardio-Renal Medicine and Hypertension, Nagoya City University, Graduate School of Medicine, \\ Nagoya 467-8601, Japan \\ Correspondence should be addressed to Nobuhiro Suzumori, og.n.suz@med.nagoya-cu.ac.jp
}

Received 29 March 2012; Accepted 25 April 2012

Academic Editor: Yasuhiko Sugawara

Copyright (๑) 2012 Nobuhiro Suzumori et al. This is an open access article distributed under the Creative Commons Attribution License, which permits unrestricted use, distribution, and reproduction in any medium, provided the original work is properly cited.

Microangiopathic antiphospholipid-associated syndromes (MAPSs) are reported as encompassing several conditions mainly affecting the microvasculature of selected organs: the liver in HELLP syndrome (hemolysis, elevated liver enzymes, and low platelet); kidney, brain, and skin in TTP (thrombotic thrombocytopenic purpura). It is predominant in patients with catastrophic antiphospholipid syndrome (APS). A recent report suggests that APS is not only a thrombotic disease but also associated with microangiopathic features, and it can explain the greater prevalence of HELLP syndrome in these patients. We here report a case of MAPS during pregnancy associated with systemic lupus erythematosus (SLE) in early second trimester.

\section{Introduction}

Obstetrical complications in revised classification criteria for the antiphospholipid syndrome (APS) include three or more unexplained spontaneous abortions before 10 weeks of gestation, intrauterine fetal death, and one or more premature births of a morphologically normal neonate before 34 weeks of gestation with continuous antiphospholipid (aPL) antibodies present in plasma [1]. APS is clearly related to maternal morbidity, and it is known to be a major cause of fetal loss due to a thrombotic tendency leading to placental infarction during pregnancy [2].

A recent report suggests that APS is not only a thrombotic disease but also associated with microangiopathic features, and it can explain the greater prevalence of HELLP (hemolysis, elevated liver enzymes, and low platelet count) syndrome in these patients [3]. Hepatic infarction, retinal vascular occlusions, and deep venous thrombosis (DVT) have now been reported in patients with HELLP syndrome [4]. Microangiopathic antiphospholipid-associated syndromes (MAPSs) are proposed as encompassing several conditions mainly affecting the microvasculature of selected organs: the liver in HELLP syndrome; kidney, brain, and skin in TTP (thrombotic thrombocytopenic purpura) and disseminated intravascular coagulation (DIC) [5].

Among HELLP cases diagnosed antepartum, $90 \%$ are in the third trimester and the syndrome rarely occurs before 27 weeks of gestation [6]. However, it is wellknown that several patients with APS suffer HELLP syndrome at very early onset despite low-dose aspirin and heparin combined therapy [7]. We here report a case of MAPS during pregnancy associated with systemic lupus erythematosus (SLE) in early second trimester.

\section{Case Report}

A 39-year-old Japanese woman with a history of one miscarriage at 6 weeks' gestation had become pregnant under anticoagulation treatment of $2500 \mathrm{U}$ danaparoid sodium because of her osteoporosis and osteonecrosis of femoral head and 
$100 \mathrm{mg}$ aspirin per day from 4 weeks' gestation at Nagoya City University Hospital. She has had a 22-year history of SLE, which had been in remission for more than 4 years under a treatment of $5 \mathrm{mg}$ prednisolone daily.

At this point, our case was not diagnosed with APS, because she suffered from only one miscarriage. However, we planed to do anticoagulant treatment during pregnancy, because her lupus anticoagulant (LA) [8] was ascertained to be strongly positive by diluted activated partial thromboplastin time (aPTT) methods and diluted Russel's viper venom time (RVVT) before pregnancy. Prednisolone therapy was interrupted before this pregnancy because the patient thought it affected infertility.

Her edema and protein urea appeared at 17 weeks' gestation (Table 1). Her blood pressure was $121 / 78 \mathrm{mmHg}$ and laboratory studies showed anti- $\beta 2$-glycoprotein I ( $\beta 2 \mathrm{GPI})$ $9.4 \mathrm{U} / \mathrm{mL}$ (normal range $<1.9$ ), aPTT $30 \%$, LA by aPTT 30 seconds, LA by RVVT 1.47, and urinary protein $2.0 \mathrm{~g} /$ day at 17 weeks of gestation. At 21 weeks of gestation, she had body edema and protein urea $(8.1 \mathrm{~g} / 24 \mathrm{~h})$. Laboratory studies were shown in Table 1. At 21 weeks of gestation she was hospitalized because of severe edema and acute renal dysfunction, and treatment of $40 \mathrm{mg}$ prednisolone daily started at 22 weeks of gestation.

She had developed epigastralgia and vomiting. Thrombocytopenia and hemolytic anemia together with liver and renal dysfunction and an elevated level of C-reactive protein were observed (Table 1). At 22 weeks and 6 days of gestation, emergency cesarean section was performed because of fetal distress with loss of variability and severe late deceleration, abnormal Doppler flow velocimetry waveform analysis suggestive of fetal hypoxemia, and the HELLP syndrome. The baby was weighting $414 \mathrm{~g}$, and the clinical manifestations were morphologically normal. Four days after birth the baby died of pulmonary hemorrhage. Placental infarction was pathologically found.

Her laboratory examinations showed liver dysfunction, and the data from coagulation studies indicated the diagnostic criteria for DIC. Intravascular infusion of nafamostat methylate together with antibiotics, gamma-globulin $15 \mathrm{~g}$, and platelet transfusion gradually improved the clinical symptoms and laboratory abnormalities. The patient had no fever or persistent epigastralgia. Her blood pressure was $140 / 92 \mathrm{mmHg}$ and pulse was 118 per min under antihypertensive therapy. Her renal biopsy showed lupus nephritis INS/RPS class IV with mesangial interposition, massive subendothelial, subepithelial and mesangial deposit, fibrinoid necrosis, karyorrhexis, swelling of endothelial cells, and foam cells. The patient was diagnosed MAPS. Then multitarget therapy (prednisolone, cyclosporine, and mizoribine) was started.

Presently, the patient is now being treated as an outpatient who is asymptomatic, while continuing treatment with cyclosporine A $(75 \mathrm{mg} /$ day $)$, mizoribine $(100 \mathrm{mg} /$ day $)$, and prednisolone $(25 \mathrm{mg} /$ day $)$. Anticoagulation with warfarin ( $2 \mathrm{mg} /$ day) was started to a target international normalized ratio (INR) of 2-3.

\section{Discussion}

MAPS have been reported as encompassing several conditions mainly affecting the microvasculature of selected organs: the liver in HELLP syndrome, kidney, brain and skin in TTP and DIC. The present case had HELLP syndrome, renal dysfunction, and DIC.

Several lines of evidence suggest that MAPS may be triggered, by mainly infections, trauma, surgery, anticoagulation withdrawal, malignancies, or lupus flare, or infrequently appear during pregnancy or puerperium $[9,10]$. Pauzner et al. reported the relationship between liver infarction in HELLP syndrome and APS and indicated that hepatic infarction during pregnancy was almost always associated with APS [4]. Electronic microscope findings of her renal biopsy demonstrated evidence of microangiopathic, although we could not find liver infarction in the present case.

The term thrombotic microangiopathic haemolytic anaemia (TMHA) was originally introduced in 1952 [11]. TMHA encompasses TTP and hemolytic-uremic syndrome (HUS). Espinosa et al. reviewed the association of aPL and TMHA comprehensively [12]. Simultaneous with the TMHA and aPL story came the association of patients with HELLP syndrome and aPL [13]. However, in the HELLP syndrome, hepatic infarctions are not uncommonly documented and are undoubtedly due to small vessel perturbation [13]. In our present case of MAPS with the HELLP syndrome, there were virtually no large vessel occlusions.

The APS has the multifactorial pathogenesis though the criteria include arterial, venous, or small vessel thrombosis [1]. The first major player in MAPS might be the endothelial cells. Endothelial cell activation has been demonstrated in TTP, aPL and HELLP syndrome [14-17]. The pathogenicity of some aPL has been dramatically demonstrated in animal models [14]. The many complex pathways were involved in intracellular signaling resulting in the conversion of cells, particularly endothelial, to a prothrombotic state $[15,16]$. The influence of these antibodies on monocytes [18], as well as on platelets antibodies is now also well described [19]. The aPL, are known to be heterogeneous in function and specificity and that more than one type may be present in any individual with APS. It is clear that APS is a multiorgan-multisystem disease with multiple possible clinical manifestations.

The aspirin and heparin combined therapy is effective in $70-80 \%$ of recurrent fetal loss patients with APS [20]. Our present case was treated with danaparoid instead of heparin to avoid osteoporosis because the patient experienced osteonecrosis of femoral head. Magnani HN reported an analysis of clinical outcomes of 91 pregnancies treated with danaparoid in 83 women with a history of thrombophilia and/or repeated pregnancy loss [21]. The successful birth rate and adverse event profile indicated that danaparoid can be an effective and safe alternative antithrombotic in pregnancies complicated by intolerance or resistance to low molecular-weight heparins.

Among HELLP cases diagnosed antepartum, 90\% are in the third trimester, and the syndrome rarely occurs before 27 weeks of gestation [6]. Early onset of HELLP syndrome 
TABLE 1: Laboratory data (abnormal findings are bold).

\begin{tabular}{|c|c|c|c|c|c|}
\hline & 17W5D & 21W1D & $22 \mathrm{~W} 6 \mathrm{D} \mathrm{C} / \mathrm{S}$ & Postpartum (Day 14) & Normal range \\
\hline \multicolumn{6}{|l|}{ Blood count } \\
\hline $\mathrm{WBC}(\mu \mathrm{L})$ & 5200 & 6100 & 5600 & 5000 & $3000-8500$ \\
\hline $\mathrm{RBC}(\mu \mathrm{L})$ & 2.87 & 2.65 & 2.51 & 2.07 & $3.78-4.99 \times 10^{6}$ \\
\hline $\mathrm{Hb}(\mathrm{g} / \mathrm{dL})$ & 9.2 & 8.6 & 7.9 & 6.5 & $10.8-14.9$ \\
\hline $\mathrm{Ht}(\%)$ & 27.3 & 25.6 & 23.4 & 19.9 & $35.6-45.4$ \\
\hline Plt $(\mu \mathrm{L})$ & 168 & 144 & 55 & 133 & $150-361 \times 10^{3}$ \\
\hline \multicolumn{6}{|l|}{ Coagulation test } \\
\hline aPTT (\%) & & 48.6 & 49.6 & 94.2 & $76-130$ \\
\hline PT (\%) & & 136.5 & 134.2 & 134.2 & $70-130$ \\
\hline Fibrinogen (mg/dL) & & 441 & 414 & 206 & $200-400$ \\
\hline $\mathrm{FDP}(\mu \mathrm{g} / \mathrm{mL})$ & & 12.6 & 38.5 & 29.9 & $<5.0$ \\
\hline $\mathrm{D}$-dimer $(\mu \mathrm{g} / \mathrm{mL})$ & & 6.6 & 24.5 & 22.1 & $<1.0$ \\
\hline \multicolumn{6}{|l|}{ Immunological data } \\
\hline $\mathrm{CRP}(\mathrm{mg} / \mathrm{dL})$ & $<0.3$ & 0.54 & 1.78 & $<0.3$ & $<0.30$ \\
\hline C3 (mg/dL) & 55 & 36 & 39 & 35 & $68-128$ \\
\hline C4 (mg/dL) & 14 & 16 & 17 & 17 & $13-36$ \\
\hline CH50 (U/mL) & 37.2 & 28.4 & 29.3 & 33.5 & $32.0-48.0$ \\
\hline Anti-dsDNA Ab (IU/mL) & 50 & 38 & 43 & 28 & $<12$ \\
\hline Anti-ssDNA Ab (AU/mL) & & 560 & 800 & 412 & $<25$ \\
\hline Anti-Sm Ab (index) & $<5.0$ & $<5.0$ & $<5.0$ & $<5.0$ & $<7.0$ \\
\hline ANA (index) & 1280 & 640 & 640 & & $<40$ \\
\hline \multicolumn{6}{|l|}{ Serum chemistry } \\
\hline $\mathrm{TP}(\mathrm{g} / \mathrm{dL})$ & 5.5 & 4.4 & 3.9 & 3.7 & $6.7-8.3$ \\
\hline AST (IU/L) & 20 & 27 & 165 & 20 & $13-33$ \\
\hline ALT (IU/L) & 11 & 8 & 70 & 9 & $6-27$ \\
\hline LDH (IU/L) & 260 & 268 & 495 & 343 & $119-229$ \\
\hline ALP (IU/L) & 210 & 232 & 297 & 200 & $115-359$ \\
\hline T-Bil (mg/dL) & 0.4 & 0.4 & & & $0.3-1.2$ \\
\hline BUN (mg/dL) & 18 & 41 & 56 & 30 & $8-22$ \\
\hline Creatinine (mg/dL) & 0.8 & 1.9 & 3.3 & 2.3 & $0.4-0.7$ \\
\hline Uric acid (mg/dL) & 7.3 & 11.3 & 11.3 & 9.6 & $2.3-7.0$ \\
\hline Blood pressure (mmHg) & $121 / 78$ & $150 / 80$ & $160 / 95$ & $154 / 84$ & \\
\hline \multicolumn{6}{|l|}{ Urine } \\
\hline Urinary protein (g/day) & 2 & 8.1 & 4.4 & 9.1 & $0.03-0.12$ \\
\hline Urinary sugar & Negative & Negative & Negative & Negative & \\
\hline
\end{tabular}

and severe organ dysfunction are the characteristics of the treatment failure of obstetrical APS. Target therapy not for thrombosis but for microangiopathy is needed for patients with APS against anticoagulant therapy.

\section{References}

[1] S. Miyakis, M. D. Lockshin, T. Atsumi et al., "International consensus statement on an update of the classification criteria for definite antiphospholipid syndrome (APS)," Journal of Thrombosis and Haemostasis, vol. 4, no. 2, pp. 295-306, 2006.

[2] R. H. W. M. Derksen, M. A. Khamashta, and D. W. Branch, "Management of the obstetric antiphospholipid syndrome," Arthritis and Rheumatism, vol. 50, no. 4, pp. 1028-1039, 2004.

[3] S. Appenzeller, F. H. Souza, A. Wagner Silvia de Souza, Y. Shoenfeld, and J. F. de Carvalho, "HELLP syndrome and its relationship with antiphosphplipid syndrome and antiphospholipid antibodies," Seminars in Arthritis and Rheumatism, vol. 41, pp. 517-523, 2011.

[4] R. Pauzner, M. Dulitzky, H. Carp et al., "Hepatic infarctions during pregnancy are associated with the antiphospholipid syndrome and in addition with complete or incomplete HELLP syndrome," Journal of Thrombosis and Haemostasis, vol. 1, no. 8, pp. 1758-1763, 2003.

[5] R. A. Asherson, S. Pierangeli, and R. Cervera, "Microangiopathic antiphospholipid-associated syndromes revisitednew concepts relating to antiphospholipid antibodies and syndromes," Journal of Rheumatology, vol. 34, no. 9, pp. 17931795, 2007.

[6] T. M. Rahman and J. Wendon, "Severe hepatic dysfunction in pregnancy," Monthly Journal of the Association of Physicians, vol. 95, no. 6, pp. 343-357, 2002. 
[7] Y. Wada, Y. Sakamaki, D. Kobayashi et al., "HELLP syndrome, multiple liver infarctions, and intrauterine fetal death in a patient with systemic lupus erythematosus and antiphospholipid syndrome," Internal Medicine, vol. 48, no. 17, pp. 1555$1558,2009$.

[8] M. Ogasawara, K. Aoki, E. Matsuura, H. Sasa, and Y. Yagami, "Anti $\beta 2$ glycoprotein I antibodies and lupus anticoagulant in patients with recurrent pregnancy loss: prevalence and clinical significance," Lupus, vol. 5, no. 6, pp. 587-592, 1996.

[9] J. A. Gómez-Puerta, J. Sanin-Blair, and C. GalarzaMaldonado, "Pregnancy and catastrophic antiphospholipid syndrome," Clinical Reviews in Allergy and Immunology, vol. 36, no. 2-3, pp. 85-90, 2009.

[10] W. Branch, "Report of the obstetric APS task force: 13th international congress on antiphospholipid antibodies, 13th April 2010," Lupus, vol. 20, no. 2, pp. 158-164, 2011.

[11] W. S. Symmers, "Thrombotic microangiopathic haemolytic anaemia (thrombotic microangiopathy)," British Medical Journal, vol. 2, no. 4790, pp. 897-903, 1952.

[12] G. Espinosa, S. Bucciarelli, R. Cervera et al., "Thrombotic microangiopathic haemolytic anaemia and antiphospholipid antibodies," Annals of the Rheumatic Diseases, vol. 63, no. 6, pp. 730-736, 2004.

[13] D. Le Thi Thuong, N. Tieulié, N. Costedoat et al., "The HELLP syndrome in the antiphospholipid syndrome: retrospective study of 16 in 15 women," Annals of the Rheumatic Diseases, vol. 64, no. 2, pp. 273-278, 2005.

[14] S. S. Pierangeli, M. Colden-Stanfield, X. Liu, J. H. Barker, G. L. Anderson, and E. N. Harris, "Antiphospholipid antibodies from antiphospholipid syndrome patients activate endothelial cells in vitro and in vivo," Circulation, vol. 99, no. 15, pp. 19972002, 1999.

[15] S. S. Pierangeli, R. G. Espinola, X. Liu, and E. N. Harris, "Thrombogenic effects of antiphospholipid antibodies are mediated by intercellular cell adhesion molecule-1, vascular cell adhesion molecule-1, and P-selectin," Circulation Research, vol. 88, no. 2, pp. 245-250, 2001.

[16] P. L. Meroni, E. Raschi, C. Testoni, and M. O. Borghi, "Endothelial cell activation by antiphospholipid antibodies," Clinical Immunology, vol. 112, no. 2, pp. 169-174, 2004.

[17] J. J. J. Hulstein, P. J. van Runnard Heimel, A. Franx et al., "Acute activation of the endothelium results in increased levels of active von Willebrand factor in hemolysis, elevated liver enzymes and low platelets (HELLP) syndrome," Journal of Thrombosis and Haemostasis, vol. 4, no. 12, pp. 2569-2575, 2006.

[18] H. Zhou, A. S. Wolberg, and R. A. S. Roubey, "Characterization of monocyte tissue factor activity induced by IgG antiphospholipid antibodies and inhibition by dilazep," Blood, vol. 104, no. 8, pp. 2353-2358, 2004.

[19] M. E. Vega-Ostertag, E. N. Harris, and S. S. Pierangeli, "Intracellular events in platelet activation induced by antiphospholipid antibodies in the presence of low doses of thrombin," Arthritis and Rheumatism, vol. 50, no. 9, pp. 2911-2919, 2004.

[20] D. W. Branch, M. Gibson, and R. M. Silver, "Recurrent miscarriage," The New England Journal of Medicine, vol. 363, no. 18, pp. 1740-1747, 2010.

[21] H. N. Magnani, "An analysis of clinical outcomes of 91 pregnancies in 83 women treated with danaparoid (Orgaran)," Thrombosis Research, vol. 125, no. 4, pp. 297-302, 2010. 


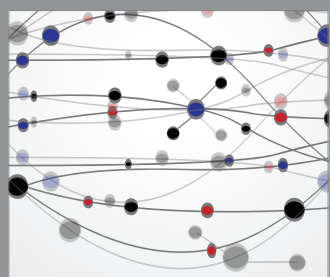

The Scientific World Journal
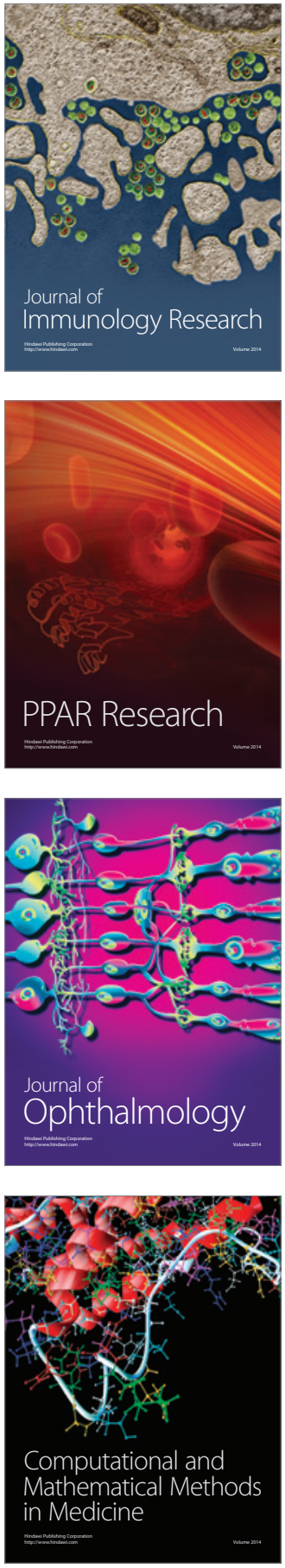

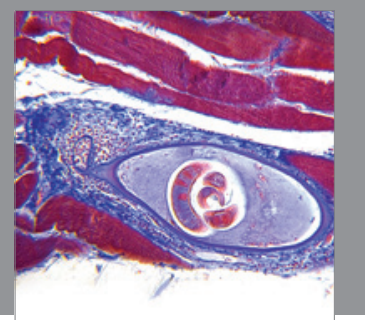

Gastroenterology

Research and Practice
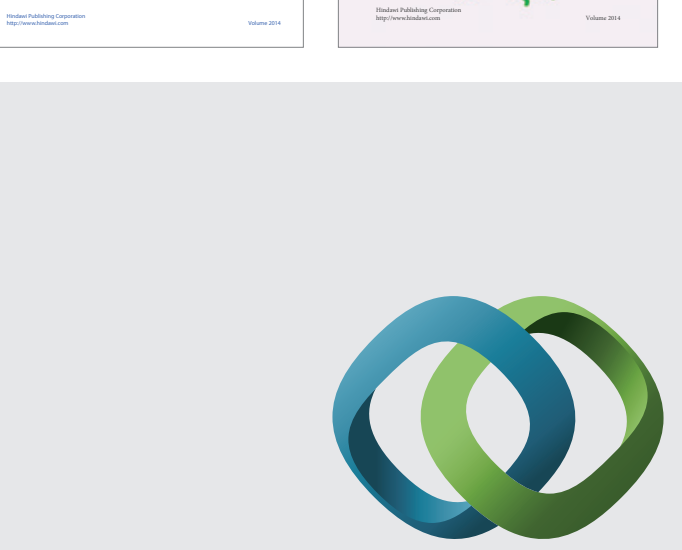

\section{Hindawi}

Submit your manuscripts at

http://www.hindawi.com
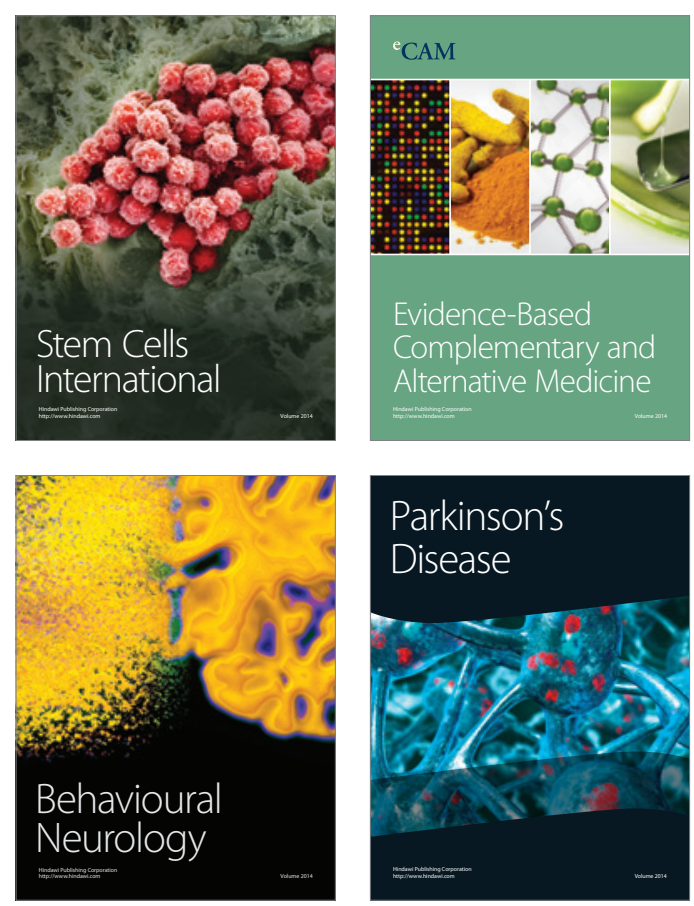

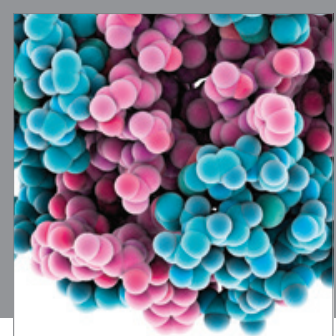

Journal of
Diabetes Research

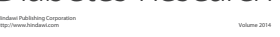

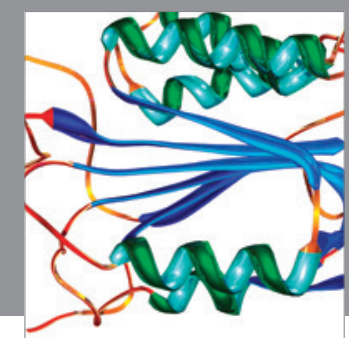

Disease Markers
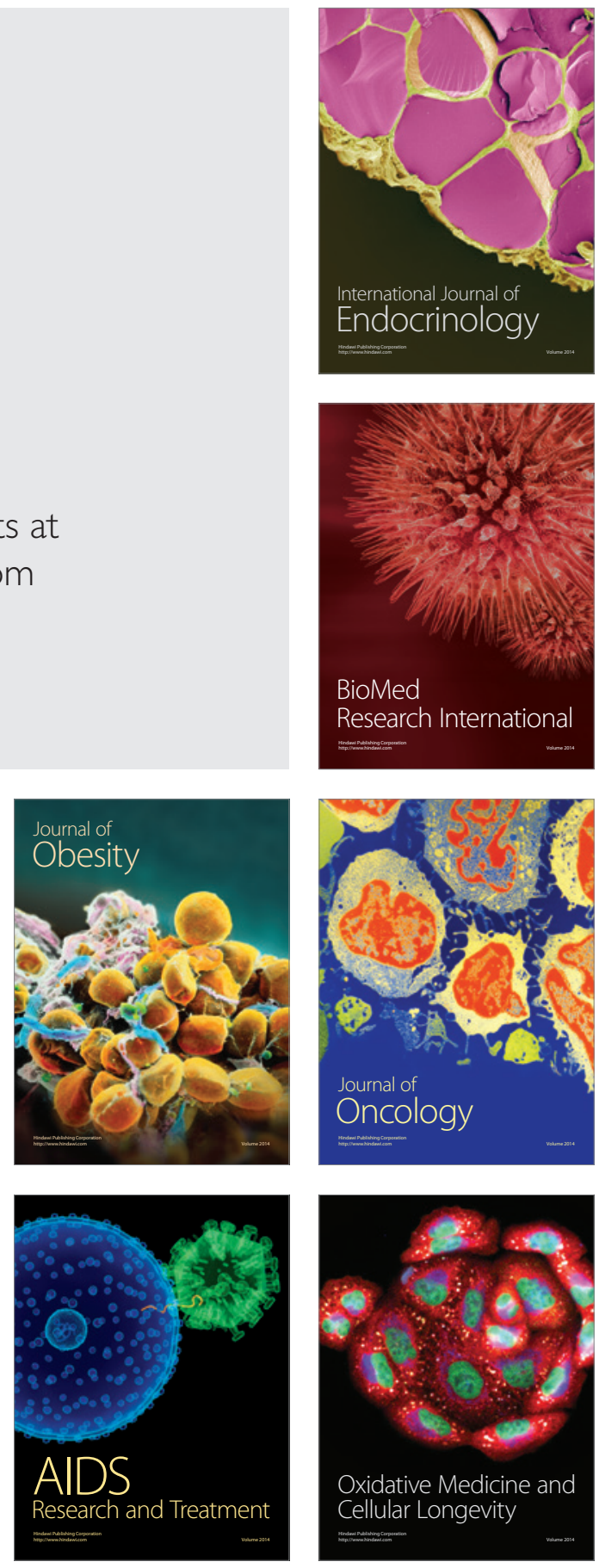